\title{
IMPLEMENTACIÓN DE HERRAMIENTAS PARA EL DIAGNÓSTICO DE INNOVACIÓN EN UNA EMPRESA DEL SECTOR CALZADO EN COLOMBIA
}

\author{
Bibiana Arango Alzate \\ Ph.D en Tecnología de Recursos Forestales de la Universidad de Sao Paulo - USP \\ Docente de pregrado y postgrado en temas de gestión de la tecnología y la innovación de la Escuela de \\ Ingenierías de la Universidad Pontificia Bolivariana - UPB \\ bibiana.arango@upb.edu.co (Bolívia)
}

\section{Jennifer Betancourt Hurtado}

Estudiante Universidad de Ingeniería Industrial de la Universidad Pontificia Bolivariana - UPB jeniferb42@hotmail.com (Bolívia)

\section{Luisa Fernanda Martinez Lopez}

Estudiante Universidad de Ingeniería Industrial de la Universidad Pontificia Bolivariana - UPB luisafernanda.martinezlo@alfa.upb.edu.co (Bolívia)

\section{RESUMEN}

En el presente trabajo se realizó un análisis de la innovación y la tecnología en una empresa del sector calzado, dedicada a producir y comercializar toda clase de calzado plástico y en tela para dama, hombre y niño. La producción de calzados de materiales sintéticos como el PVC, tiene aplicaciones en muchos mercados, y ofrece amplias posibilidades para que una organización sea internacionalmente competitiva. Es por esto que la empresa se ve en la necesidad de realizar un análisis o medición actual de sus recursos tecnológicos donde se emplean herramientas de diagnóstico de innovación (Albacete, Catalán y MTG) dando a conocer el estado actual de la empresa en su perfil tecnológico e innovador, evaluando su situación competitiva, la gestión y las capacidades de innovación, posteriormente a esto se hizo un análisis de los resultados obtenidos por las herramientas, donde se identificaron las fortalezas y las oportunidades que debe aprovechar la empresa para mejorar a nivel tecnológico e innovador, de otra forma, crear un diferenciador en estos aspectos mejorando el desempeño de la empresa en el mercado y lograr ser más competitiva en el mercado.

Palabras Claves: Innovación; Tecnología; Herramientas de diagnóstico; Competitividad. 


\section{INTRODUCCIÓN}

Hoy en día los procesos de innovación son factores claves dentro de las organizaciones ya que sin importar el sector productivo al cual pertenezcan, estos son los que le permiten lograr altos niveles de competitividad y una permanecía en el entorno. Las cambiantes necesidades del mercado y la agresiva competencia son los desafíos a los que se enfrentan las organizaciones de hoy.

Por este motivo es necesario gestionar diferentes variables dentro de las cuales se encuentra la tecnología, la cual se convierte en un elemento determínate y diferenciador para las organizaciones. Según (Fonseca et al., 2011), la gestión de esta variable ha proporcionado diferentes herramientas, siendo una de estas el diagnóstico tecnológico, cuya función es evaluar los recursos disponibles identificando las fortalezas y debilidades que posee una empresa con respecto a la tecnología, de esta manera se orientara ese conocimiento a la creación de estrategias u oportunidades para la generación de valor dentro de los procesos de la organización para ser más competitivos.

Por lo anterior, este trabajo pretende realizar un diagnóstico tecnológico con el fín de identificar los factores y aspectos que deben monitorearse y mejorarse para generar innovación dentro de la empresa, teniendo en cuenta que esta es la clave para el crecimiento económico, la competitividad y la productividad. Este diagnóstico se hará mediante el uso de las herramientas de medición tecnológica TEST CATALAN, ALBACETE y MTG (Metodología de Gestión Tecnológica por Proyectos en la Organización).

\section{REVISIÓN LITERARIA}

\subsection{Sobre el Calzado}

"La industria del calzado se caracteriza por ser una de las actividad que ha mantenido unos niveles de estabilidad a lo largo del tiempo" (Ballón, 2008)

\subsection{Producción del calzado}

Revista de Administração e Inovação, São Paulo, v. 12, n.3 p. 310-329, jul./set. 2015. 
En los últimos años la entrada (China e Indonesia) y salida (Argentina, Alemania, Francia y Estados Unidos) de nuevos competidores, están haciendo que el sector calzado sea más atractivo y a su vez más competitivo. (Herrera, Hortúa \& Morales, 2009).

Con el fin de estimar los recursos disponibles en las organizaciones en términos de tecnologías, procesos y conocimientos, el diagnóstico tecnológico ha sido utilizado en diversas organizaciones con el objetivo de orientar los procesos de planeación y la definición de estrategias para mantener o mejorar su posición competitiva en los entornos donde se desenvuelven (Fonseca et al., 2011).

La gestión tecnológica y la innovación se han convertido en los constituyentes claves en el entorno empresarial para garantizar el crecimiento, la permanencia en el tiempo, enfrentarse a las situaciones cambiantes actuales y de futuro, generar avance tecnológico y dar mayor valor agregado tanto a la organización como a sus clientes (Zartha, Orozco, Vergara \& Jessie, 2011).

\subsection{Tecnología del calzado en el mundo}

La innovación tecnológica y la calidad del sector calzado se enfocan en el cuidado de la salud y bienestar del usuario, marcas que han logrado hacerse un espacio en el mercado basando su estrategia promocional en su tecnología beneficiosa para la salud, como lo menciona en Recinos (2011) la Gerente de Inteligencia competitiva Marta Olivia Recinos del Ministerio de Economía de El Salvador, un ejemplo es la empresa Masai Barefoot Technology (MBT) que ofrece un zapato que procura la postura correcta para la espina dorsal al caminar. También estas industrias están realizando zapatos personalizados y hechos a la medida, que satisfagan las necesidades emocionales, por ejemplo LaCrosse Footwear que creó un programa de calzado personalizado llamado "myDanner" para crear botas únicas y personalizadas, o la empresa Zazzle donde el cliente pueda expresarse artísticamente, y seleccionar el color de sus zapatos. (p.17)

De acuerdo con CSM3D International Limited (2011), las empresas están enfrentando retos para incrementar su competitividad, y para ello se están invirtiendo en software que permitan elaborar versiones virtuales de sus modelos en 3D logrando reducir los costos y tiempos para el desarrollo y el lanzamiento de nuevos productos; Por otro lado Recinos(2011) señala que la compañía Under Armour que produce prendas de vestir deportivas, incursionó en el segmento de calzado deportivo con la ayuda de la tecnología para elaborar un prototipo rápido. (p.18)

Según Salinas \& Angulo (2001) el concepto de automatización y racionalización avanza a pasos agigantados dentro de las principales operaciones que comprende la fabricación de calzados. Así sea en la fabricación integral del zapato, como en la de sus componentes, la incursión de robots es cada

Revista de Administração e Inovação, São Paulo, v. 12, n.3 p. 310-329, jul./set. 2015. 
vez frecuente. Así mismo Salinas \& Angulo (2001) expresa que una de las empresas a la vanguardia en materia de robótica aplicada a calzados es la alemana Desma, quien propone novedosos software para la programación de funciones para robots, que hacen a la efectividad y calidad del trabajo.

El informe de conclusiones obtenidas por el primer taller nacional de tecnologías aplicadas al sector calzado Fundetec y Anetcom (2007) dio a conocer que el sector calzado dispone de una implantación muy positiva de las tecnologías desde el punto de vista de acceso a las mismas. No obstante, el sector calzado debe mejorar el grado de uso de las tecnologías de la información y comunicación (TIC) e incrementar la operatividad de las tecnologías en sus procesos de negocio. Por ejemplo de acuerdo con MUNDIPRESS (2013) el Centro Tecnológico del Calzado de la Rioja (CTCR) ha logrado destacar por su actividad en el desarrollo y control de proyectos de $\mathrm{I}+\mathrm{D}+\mathrm{i}$, así como en el servicio, en diseño del producto, moda y prototipado, la organización de propuestas formativas, la ejecución de acciones medioambientales, la investigación en nanotecnología, los avances en TIC, etc. En definitiva, las TIC ofrecen más herramientas que permiten no solo ahorros, sino la posibilidad de ser mucho más flexibles y eficientes.

\subsection{La industria del calzado en Colombia}

El Sector del calzado en Colombia, está conformado por las empresas que fabrican, importan, comercializan y exportan, los diferentes tipos de calzado, entre los que se destaca el calzado deportivo, con una participación cercana al $40 \%$ del total de la producción nacional, seguido por botas, botas para dama, calzado casual para hombre y dama, zapatillas sintéticas y calzado para niño. (Instituto Español de Comercio Exterior , 2005, p.8)

"El sector se caracteriza por una fuerte competencia entre productores nacionales recrudecida por las importaciones y por los altos volúmenes de contrabando (en su mayoría procedentes de China)". (ICEX , 2005, p.10)

Desde el 2006, la Asociación Colombiana de Calzado ha estado ejerciendo presión para bloquear las importaciones ilegales del calzado manufacturado en China; sin embargo, estas iniciativas no han tenido el resultado esperado. Incluso las campañas dirigidas al público para promocionar el calzado nacional han quedado sin efecto, debido a que los consumidores no pueden detectar el origen de los productos; los compradores simplemente demandan precios más bajos y la lealtad a las marcas no es algo que les interese (Recinos, 2011, p.10)

Revista de Administração e Inovação, São Paulo, v. 12, n.3 p. 310-329, jul./set. 2015. 
Por otro lado según Ortega \& Hurtado (2012) en Colombia en la produccion de calzado, el tema innovación en los pequeños empresarios es prácticamente nulo, pues mas allá de identificar, conocer que es lo está de moda y los modelos que más está consumiendo la población, no existe una cultura de transferencia de conocimiento de los empresarios hacia los colaboradores, muchos de los procesos se realizan de manera informal, los trabajadores participan muy poco aportando ideas, la maquinaria usada en la producción en promedio tiene más de 12 años antigüedad, no existe ninguna persona que se encargue del conocimiento y la innovación y en general el pequeño empresario, conoce muy poco del tema innovación y lo que se hace es más el resultado del conocimiento empírico que el de una política y una culturización con el tema. (p.29)

Pensando en la importancia del desarrollo tecnológico del sector del calzado, Ceinnova que es el Centro de Desarrollo Tecnológico para las Industrias del Calzado, Cuero y Afines de Colombia (2009), y cuya actividad fundamental es la prestación de servicios a las empresas del sector, en transferencia de tecnología, información técnica y de moda, mejoramiento de la calidad, asistencia técnica, diseño y desarrollo de productos, pruebas y ensayos de laboratorio y desarrollo empresarial; Ha desarrollado un programa a través del cual busca determinar las necesidades tecnológicas competitivas para las empresas del sector, este programa cuenta con los siguientes módulos:

- Determinación de las necesidades tecnológicas conforme a las estrategias de la empresa y con base en los factores de competitividad empresarial

- Soporte para la determinación de las tecnologías medulares y complementarias requeridas

- Determinación de proyectos de desarrollo tecnológico

\section{LA INNOVACIÓN TECNOLÓGICA}

La innovación es el proceso de convertir ideas en productos o servicios nuevos o mejorados, que aporten un rendimiento al mercado y unos beneficios a la empresa objeto del proceso innovador. Cuando se habla de innovación no se está haciendo referencia exclusivamente a cuestiones relacionadas con la tecnología, sino que también se incluyen aspectos relacionados con la organización y las formas de comercialización de los bienes, entre otras. (Cámara Oficial de Comercio de Madrid, 2004, p.4)

Según OECD y EUROSTAT(2005) el Manual de Oslo se define la innovación como la introducción de un nuevo, o significativamente mejorado, producto (bien o servicio), de un nuevo

Revista de Administração e Inovação, São Paulo, v. 12, n.3 p. 310-329, jul./set. 2015. 
método de comercialización o de un nuevo método organizativo, en las prácticas internas de la empresa, la organización del lugar de trabajo o las relaciones externas. Para que haya innovación, hace falta como mínimo que el producto, el proceso, el método de comercialización o el método de organización sean nuevos (o significativamente mejorados) para la empresa.

El Manual de Innovación de Movimiento Brasil Competitivo (MBC) define la innovación tecnológica como "el resultado de aplicar los conocimientos adquiridos a través de la investigación científica se aplica a los productos o procesos de producción, con nuevas características y eficaces aumento de la productividad y la calidad, lo que resulta en una mayor competitividad". (Confederação Nacional da Indústria, 2010, p.19)

\subsection{Características de la innovación}

De acuerdo con CNI(2010) algunas de las características de la innovación son:

- El riesgo de fracaso está siempre presente cuando busca innovar

- Las personas creativas son la materia prima básica en el proceso de generación de innovaciones. Apreciar los talentos

- Ambientes adecuados pueden generar ideas creativas e innovaciones

- La innovación es un proceso abierto

- La innovación se puede conseguir de diferentes maneras, incluyendo mediante el uso de métodos estructurados

- La innovación es un fenómeno que ocurre en todas las áreas del conocimiento, como en los negocios, en donde:

$>\quad$ La innovación debe estar alineada con la estrategia

$>\quad$ La mayoría de las innovaciones provienen de tres fuentes básicas: clientes, competidores y empleados

$>\quad$ La innovación es un fenómeno que ocurre en el mercado

$>\quad$ Innovar es crear valor para la empresa. (p.20)

\subsection{Ciclo de innovación}

Según CNI (2010) por regla general, en los negocios, las innovaciones son fenómenos complejos y pasan por un ciclo que involucra a su surgimiento, crecimiento, madurez y declive. Cada 
una de estas etapas implica una serie de pasos que pueden requerir de actividades tales como la investigación, el desarrollo, la creación de prototipos, producción, distribución, comercialización, suministro, asistencia técnica post-venta, marketing y gestión de marcas. (p.21)

\subsection{La innovación y la competitividad}

La innovación y la competitividad se asocian directamente con el desempeño financiero y el éxito del negocio. En la economía globalizada del siglo XXI, en el que la competencia es global, la innovación es un factor de competitividad fundamental, tan importante como la calidad de los productos y de servicios clientes y más importante que la reducción de costes y precios. (Mattos et al., 2008, p.8).

Marco conceptual sobre la doble relación entre competitividad e innovación

“contar con empresas innovadoras supone no sólo una mayor competitividad de la economía en su conjunto, sino también la generación de derramamientos de índole tecnológicos (technology spillovers) sobre los restantes agentes económicos" (Jaramillo et al., 2001, citado en Monge \& Hewitt, 2008, p.21)

\subsection{Herramientas para la gestión de la innovación}

De acuerdo con Yoshida, Gomes, \& de Francisco (2008) La palabra "herramienta" se utiliza porque es un término simple y también por ser una expresión que indica un beneficio práctico directo, además de indicar que el usuario controla la forma en que la herramienta se debe aplicar y cómo se utiliza.(p.5). Existen muchas maneras para aumentar la producción de innovación de las organizaciones, entre ellas está la importancia del proceso de la planeación estratégica que se propone partir del conocimiento de la situación actual de la organización respecto a innovación para de esta manera detectar las brechas internas, estratégicas o respecto a la competencia, a continuación se hará una revisión de las tres herramientas que se utilizaran para realizar el presente diagnostico tecnológico.

\subsubsection{Test Catalan}

Revista de Administração e Inovação, São Paulo, v. 12, n.3 p. 310-329, jul./set. 2015. 
Es un test de innovación empresarial desarrollado por el instituto Catalán de Tecnología. Es un diagnóstico rápido de la capacidad de innovación de la empresa, consta de 20 preguntas distribuidas en las siguientes dimensiones:

- Estrategia de innovación

- Despliegue de la estrategia de innovación

- Cultura de la innovación

- Innovación en la cadena de valor

- Resultados de la innovación

"Luego de responder a las preguntas planteadas en estas dimensiones se procede a sumar el total de respuestas en sí y en no y de acuerdo a una tabla, se clasifica la capacidad de innovación en buena, excelente o insuficiente" (Ruiz \& Herrera, 2010, p.9)

\subsubsection{CEEI ALBACETE}

CEEI ALBACETE es una fundación Europea, que desarrollo una herramienta de acceso web libre, denominada “Autodiagnóistico de la Innovación”, esta tiene como objetivo fundamental evaluar el grado de innovación que tiene una empresa.

“Autodiagnóistico de la Innovación”, es considerada una herramienta de autodiagnóstico porque se sustenta en el hecho de que sea el propio empresario quien de forma autónoma, tome conciencia del grado de importancia que puede tener la innovación para su empresa, y se haga cargo de conocer las debilidades y fortalezas que tiene su empresa en cuanto a esta, de tal manera que se haga participe en el proceso de innovación de la empresa. (Centro Europeo de Empresas e Innovación, 2013)

Según Ruiz \& Herrera (2010) las ventajas de esta herramienta es que es fácil de diligenciar, no requiere mucho tiempo y los conceptos usados en cada una de las preguntas no son complejos, entrega una buena idea general de las dimensiones de innovación analizadas. Y su desventaja/reto es que la presentación de resultados no es exportable directamente. (p.9)

\subsubsection{MTG (Metodología de Gestión Tecnológica por Proyectos en la Organización)}

Según Zartha J, Quintero S (2009), citado en Ruiz Navas \& Herrera( 2010) la herramienta MGT, evalúa las dimensiones: 
$\square$ Organización

$\square$ Productos y servicios

$\square$ Mercado

$\square$ Proceso

Basándose en la definición de innovación entregada por la OCDE en el manual de OSLO. Para cada una de estos tipos de innovación se diagnostica: planeación, comunicación, recursos e inversiones. Luego de diligenciar las preguntas, el perfil muestra a manera de sumario en una tabla el resultado de las brechas detectadas en la organización y por cada tipo de innovación una gráfica radar. (p.11)

Otra herramienta muy interesante que pueden ser utilizadas es El radar de la innovación.

Esta herramienta realiza el diagnostico de la innovación basada en 12 dimensiones: oferta, plataformas, soluciones, clientes, experiencia de clientes, captación de valor, procesos, organización, Supply Chain,, presencia, redes y marca. De acuerdo con CNI( 2010) la clave está en que estas dimensiones son fáciles de comprender y ayudar a los administradores a implementar la gestión de la innovación como un proceso estructurado dentro de la empresa. (p.25)

\section{GESTIÓN DE LA TECNOLOGÍA}

Una gestión de la tecnología se puede definir como: gestión sistemática de todas las actividades dentro de la empresa con respecto a generación, adquisición, inicio de la producción, el procesamiento, la comercialización y la asimilación las tecnologías requeridas por la empresa, incluida la cooperación y las alianzas con otras instituciones, también regula las prácticas de diseño, promoción y gestión y herramientas para la captura y / o producción de información que permite a lo largo y continuo, mejorar calidad la productividad. (Saenz \& Capote, 2002, citado en Yoshida, Gomes \& de Francisco, 2008, p.3)

\subsection{Diagnostico tecnológico}

Con el fin de estimar los recursos disponibles en las organizaciones en términos de tecnologías, procesos y conocimientos, el diagnóstico tecnológico ha sido utilizado en diversas organizaciones con el objetivo de orientar los procesos de planeación y la definición de estrategias para mantener o mejorar su posición competitiva en los entornos donde se desenvuelven (Fonseca et al, 2011).

Revista de Administração e Inovação, São Paulo, v. 12, n.3 p. 310-329, jul./set. 2015. 
La gestión tecnológica y la innovación se han convertido en los constituyentes claves en el entorno empresarial para garantizar el crecimiento, la permanencia en el tiempo, enfrentarse a las situaciones cambiantes actuales y de futuro, generar avance tecnológico y dar mayor valor agregado tanto a la organización como a sus clientes. (Zartha, Orozco, Vergara \& Jessie, 2011)

\subsection{La Capacidad Tecnológica de Innovación (CTI)}

De acuerdo con Aguirre (2010) la capacidad tecnológica de innovación, puede definirse como "la gran variedad de conocimientos y habilidades necesarias que las empresas pueden adquirir, asimilar, utilizar, adaptar, cambiar y crear tecnología" p.17

Guan et al. (2006) sostuvo que las CTI no sólo depende de capacidad tecnológica, sino también en la capacidad crítica en el ámbito de la fabricación, comercialización, organización, la planificación de la estrategia, el aprendizaje y la asignación de recursos. También han definido CTI como la capacidad de siete dimensiones, a saber, capacidad de I+D, la capacidad de fabricación, capacidad de comercialización, la capacidad de explotar los recursos, capacidad de organización y capacidad estratégica. En conclusión, las CTI de una empresa se basan en múltiples criterios, que comprenden cuantitativos y cualitativos. (Aguirre, 2010, pp 18,19)

\section{METODOLOGÍA}

Para realizar el diagnostico tecnológico a la empresa, se recurrió a la aplicación de tres herramientas, la primera en aplicarse fue una herramienta de rápido diagnostico como lo es el TEST CATALAN, esta permitió ver en términos muy generales la capacidad de innovación que tenia la empresa; seguido a esto se aplico la herramienta ALBACETE, por medio de esta se hizo un diagnostico más profundo del grado de innovación que tiene la empresa, fue más profundo en el sentido de que esta herramienta va más allá de analizar la estrategia de innovación, la cultura de innovación y el papel de la innovación en la cadena de valor de la empresa, y a estos factores les integra la importancia de comprender la tecnología y el know how, de contar con un liderazgo en la manera de como el director establece las metas y prioridades de la innovación y mantiene un ambiente de trabajo innovador, hasta analizar como la empresa asegura que hay suficientes recursos humanos 
apropiados. Por último se recurrió a aplicar la herramienta de diagnóstico MTG (Metodología de Gestión Tecnológica por Proyectos en la Organización)

\section{RESULTADOS Y DISCUSIÓN}

\subsection{Test catalan}

Es una herramienta de diagóstico que permite establecer un test que evalúa las capacidades de innovación de una empresa, de acuerdo a: estrategia de innovación, cultura de la innovación, innovación en la cadena de valor y resultados de la innovación.

Al sumar todas las respuestas afirmativas se obtiene una calificación que determina o da una idea de la capacidad de la innovación en la empresa, la siguiente figura muestra las preguntas del test Catalán donde se determinan las fortalezas (puntos fuertes) y las oportunidades a mejorar (debilidades) en la organización.

\begin{tabular}{|c|c|c|c|}
\hline 1. & ESTRATEGIA DE INNOVACIÓN & SI & NO \\
\hline & $\begin{array}{l}\text { ¿La estrategia de su empresa tiene en cuenta la innovación y la } \\
\text { considera como un factor clave para su éxito? }\end{array}$ & $\mathrm{X}$ & \\
\hline & $\begin{array}{l}\text { ¿La estrategia de su organización es proactiva en materia de } \\
\text { innovación y se anticipa a los cambios que se producen en el } \\
\text { mercado y en el entorno? }\end{array}$ & & $\mathrm{X}$ \\
\hline & $\begin{array}{l}\text { ¿La dirección de la empresa está comprometida y ofrece pleno } \\
\text { apoyo a las actividades de innovación? }\end{array}$ & $\mathrm{X}$ & \\
\hline & $\begin{array}{l}\text { ¿Su organización dispone de un plan formal donde estén definidos } \\
\text { los objetivos, las acciones a llevar a cabo, los recursos y el } \\
\text { presupuesto necesarios para el desarrollo de las actividades de } \\
\text { innovación? }\end{array}$ & & $\mathrm{X}$ \\
\hline \multirow[t]{4}{*}{2.} & $\begin{array}{l}\text { DESPLIEGUE DE LA } \\
\text { ESTRATEGIA DE INNOVACIÓN }\end{array}$ & SI & NO \\
\hline & $\begin{array}{l}\text { ¿Su empresa dispone de un directivo a quien han sido asignadas las } \\
\text { responsabilidades en materia de innovación? }\end{array}$ & & $\mathrm{X}$ \\
\hline & $\begin{array}{l}\text { ¿Su empresa dedica recursos humanos, financieros y materiales } \\
\text { significativos al apartado de innovación? }\end{array}$ & & $X$ \\
\hline & ¿La innovación en su empresa contempla no sólo el desarrollo de & $\mathrm{X}$ & \\
\hline
\end{tabular}




\begin{tabular}{|c|c|c|c|}
\hline & $\begin{array}{l}\text { nuevos productos o servicios, sino también la mejora de los } \\
\text { procesos del negocio? }\end{array}$ & & \\
\hline & $\begin{array}{l}\text { ¿El diseño y desarrollo de nuevos productos (o servicios) se lleva } \\
\text { a cabo en función de las necesidades reales del mercado y de los } \\
\text { clientes de la empresa? }\end{array}$ & $\mathrm{X}$ & \\
\hline \multirow[t]{5}{*}{3.} & CULTURA DE LA INNOVACIÓN & SI & NO \\
\hline & $\begin{array}{l}\text { ¿Su empresa fomenta la creatividad y aprovecha todo el potencial } \\
\text { de sus directivos y trabajadores? }\end{array}$ & & $\mathrm{X}$ \\
\hline & $\begin{array}{l}\text { ¿Su empresa evalúa todas las ideas generadas dentro de la } \\
\text { organización y las aprovecha de forma sistemática para potenciar } \\
\text { su desarrollo? }\end{array}$ & & $\mathrm{X}$ \\
\hline & $\begin{array}{l}\text { ¿Su empresa aprovecha de forma sistemática las sugerencias y los } \\
\text { conocimientos de sus proveedores para generar mejoras e } \\
\text { innovaciones de producto o de proceso? }\end{array}$ & & $\mathrm{X}$ \\
\hline & $\begin{array}{l}\text { ¿Su empresa aprovecha de forma sistemática las sugerencias y las } \\
\text { quejas de sus clientes para generar mejoras e innovaciones de } \\
\text { producto o de proceso? }\end{array}$ & & $\mathrm{X}$ \\
\hline \multirow[t]{7}{*}{4.} & INNOVACIÓN EN LA CADENA DE VALOR & SI & NO \\
\hline & $\begin{array}{l}\text { ¿Su empresa desarrolla de forma sistemática nuevos productos y/o } \\
\text { servicios? }\end{array}$ & & $\mathrm{X}$ \\
\hline & $\begin{array}{l}\text { ¿Su empresa introduce de forma sistemática innovaciones y } \\
\text { mejoras en los procesos de producción? }\end{array}$ & & $\mathrm{X}$ \\
\hline & $\begin{array}{l}\text { ¿Su empresa introduce de forma sistemática innovaciones y } \\
\text { mejoras en la cadena de suministros (aprovisionamientos / } \\
\text { distribución) y en la logística? }\end{array}$ & & $\mathrm{X}$ \\
\hline & $\begin{array}{l}\text { ¿Su empresa introduce de forma sistemática innovaciones y } \\
\text { mejoras en las áreas de marketing y ventas? }\end{array}$ & & $\mathrm{X}$ \\
\hline & $\begin{array}{l}\text { ¿Su empresa introduce de forma sistemática innovaciones } \\
\text { mejoras en las áreas de servicios post-venta y soporte a clientes? }\end{array}$ & & $\mathrm{X}$ \\
\hline & $\begin{array}{l}\text { ¿Su empresa invierte regularmente en tecnología (maquinaria, } \\
\text { bienes de equipo, ordenadores...) para conseguir ventajas } \\
\text { competitivas? }\end{array}$ & $\mathrm{X}$ & \\
\hline \multirow[t]{4}{*}{5.} & RESULTADOS DE LA INNOVACIÓN & SI & NO \\
\hline & $\begin{array}{l}\text { ¿Considera que su empresa es más innovadora que la } \\
\text { competencia? }\end{array}$ & & $\mathrm{X}$ \\
\hline & $\begin{array}{l}\text { ¿Los ingresos actuales generados por los productos desarrollados } \\
\text { (o mejorados) en los } 3 \text { últimos años son significativos? }\end{array}$ & $\mathrm{X}$ & \\
\hline & TOTAL & 6 & 14 \\
\hline
\end{tabular}

Los resultados de aplicar esta herramienta indican que en la empresa, no se tiene una estrategia de innovación completamente definida por lo tanto esta no puede ser desplegada al máximo; un aspecto que impacta directamente en esto es la no existencia de una cultura de la innovación y la falta de integración de la innovación a la cadena de valor de la empresa; todas esta falencias dan como resultado una innovación insuficiente, por lo tanto la empresa no puede considerase como innovadora, debido a lo cual es preciso que todos los actores de las diferentes áreas de la empresa busquen unirse 
para establecer monitorias, controles y generar avances en todos los aspectos relacionados con innovación, teniendo en cuenta aquellos que están incluidos en el test y en los cuales su resultado fue negativo.

\subsection{CEEI ALBACETE}

Los resultados obtenidos al implementar la herramienta CEEI ALBACETE en la cual se muestran diferentes ítems: nuevas ideas de producto, desarrollo de producto, procesos de innovación. Tecnología y know how, mercado objetivo, liderazgo de la empresa, asignación de los recursos de la innovación y la forma como se evalúa esta. Presentando al final una tabla resumen de resultados obtenidos.

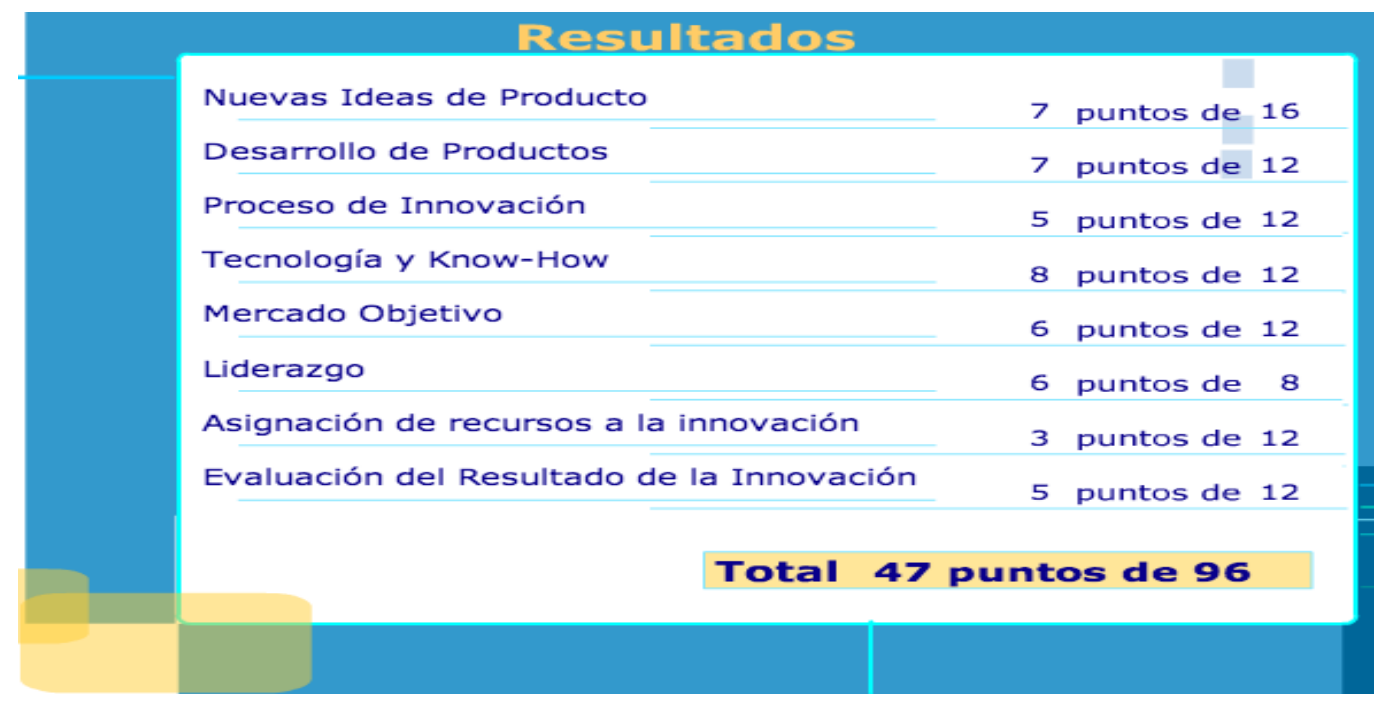

Los resultados obtenidos indican que al evaluar la capacidad de innovación de la empresa, en todas las dimensiones que analiza esta herramienta, se presentan falencias en la mayoría de los aspectos, sin embargo hay que destacar que el liderazgo que se está implementando está generando avances en cuanto a los objetivos y a la cultura de la innovación empresarial, esto último se contrapone con los resultados obtenidos en la aplicación de la herramienta anterior; por otro lado en los resultados se aprecia que la baja asignación de recursos es un factor clave para que en la empresa 
se tenga una capacidad de innovación por debajo de la media de la puntuación recomendada (96); De acuerdo con esto la empres no puede considerase como innovadora.

\subsection{MTG (Metodología de Gestión Tecnológica por Proyectos en la Organización)}

MGT es una de las herramientas de diagnóstico de la innovación más completas, esto debido a que muestra los diferentes ítems del perfil innovador y el perfil tecnológico en la empresa. La Tabla 1 es un resumen del perfil tecnológico donde se evalúan los ítems de Machine, Methods, Management y Money comparándolos con un puntaje esperado versus uno obtenido y determinando una brecha de oportunidad la cual es la diferencia de estos puntajes.

\section{Tabla 1}

Perfil Tecnológico

\begin{tabular}{|c|c|c|c|}
\hline & \multicolumn{3}{|c|}{ PUNTAJE } \\
\hline ÍTEM & Esperado & Obtenido & $\begin{array}{c}\text { Brecha de } \\
\text { oportunidad }\end{array}$ \\
\hline MACHINE & 16 & 10 & 6 \\
\hline METHODS & 20 & 10 & 10 \\
\hline MANAGEMENT & 20 & 11 & 9 \\
\hline MONEY & 20 & 10 & 10 \\
\hline Total & 76 & 41 & 35 \\
\hline
\end{tabular}

\section{Figura 1}

Brechas en el Perfil Tecnológico

Esperado Identificación de Brechas en el Perfil Tecnológico

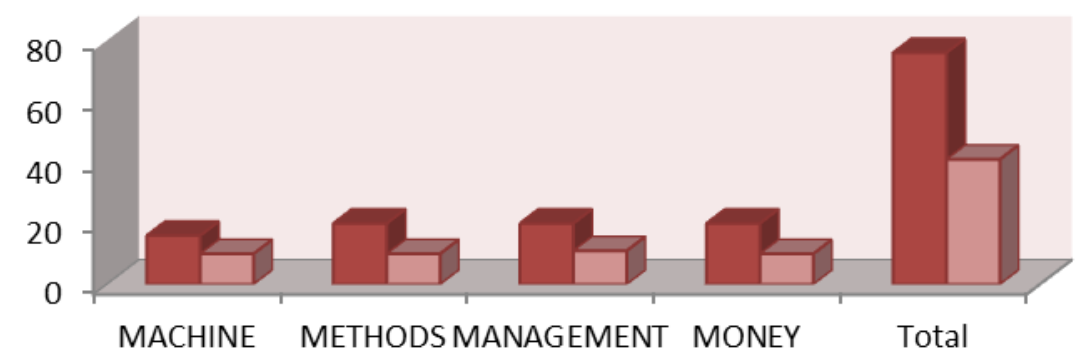

\section{Figura 2}

Segundo diagrama de brechas en el perfil tecnológico 


\section{Identificación de Brechas en el Perfil Tecnológico}

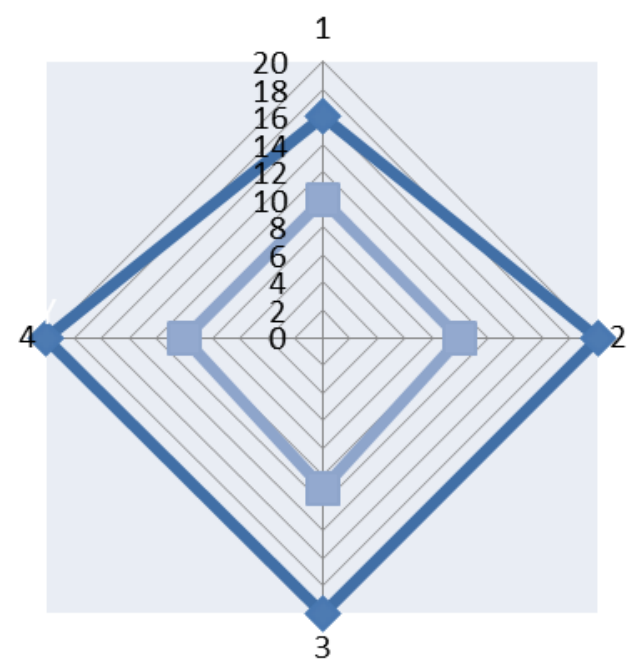

$\sim$ Esperado

$=$ Obtenido

La Figura1 muestra los vacios que se presentan en el perfil tecnológico de la empresa en las áreas de máquinas, métodos, dinero y en el manejo o administración de estos recursos.

En la Figura 2. Se puede observar que en la empresa se presenta una brecha de ocho puntos entre el valor esperado y el obtenido en maquinaria (machine); lo que indica que no cuenta con una infraestructura tecnológica buena, ya que las máquinas que tiene la empresa en los últimos años representan una tecnología básica por lo que son necesarias para la producción pero no constituye una ventaja sobre su más directa competencia. Con respecto a los métodos se obtuvo una brecha de diez puntos entre el valor esperado y el obtenido el cual es bajo, ya que la empresa tiene prioridad en generar un cambio en los productos, pero tienen falencias en la capacitación del personal en la parte del know how. En el tema de Management se tiene una brecha de nueve puntos, el motivo de esto es que la empresa se encuentra en un proceso de alineación de la gestión tecnológica con el plan estratégico y el hecho de que no se capacite el personal en el tema de gestión tecnológica afecta este resultado.

En la identificación del Dinero, se obtuvo una brecha de once puntos de diferencia entre el valor esperado y el obtenido, esto debido a que la inversión en las tecnologías transversales han contribuido muy poco al incremento de la rentabilidad de la empresa.

De la misma manera como se examinó el perfil tecnológico, las Tablas 2 y 3 son un resumen del perfil innovador de la empresa en cuanto a: organización, producto/servicio, mercado y proceso basado en la planeación, comunicación, recursos humanos e inversión realizada.

\section{Tabla 2}

Revista de Administração e Inovação, São Paulo, v. 12, n.3 p. 310-329, jul./set. 2015. 
Perfil Innovador

\begin{tabular}{|c|c|c|c|c|c|c|c|c|c|c|}
\hline \multirow{2}{*}{$\begin{array}{c}\text { Tipo de } \\
\text { Innovación }\end{array}$} & \multicolumn{2}{|c|}{ (O) ORGANIZACIÓN } & \multicolumn{2}{c|}{ (S) PRODUCTO/SERVICIO } & \multicolumn{2}{c|}{ (M) MERCADO } & \multicolumn{2}{c|}{ (P) PROCESO } & \multicolumn{2}{c|}{ Total por Item } \\
\cline { 2 - 13 } & \multicolumn{2}{|c|}{ Puntaje } & \multicolumn{2}{c|}{ Puntaje } & \multicolumn{2}{c|}{ Puntaje } & \multicolumn{2}{c|}{ Puntaje } & \multicolumn{2}{c|}{} \\
\hline Item & Esperado & Obtenido & Esperado & Obtenido & Esperado & Obtenido & Esperado & Obtenido & Esperado & Obtenido \\
\hline Planeación & 20 & 10 & 16 & 8 & 24 & 13 & 20 & 9 & 80 & 40 \\
\hline Comunicación & 12 & 6 & 12 & 5 & 20 & 11 & 0 & 0 & 44 & 22 \\
\hline Recursos & 8 & 2 & 8 & 5 & 0 & 0 & 8 & 2 & 24 & 9 \\
\hline Inversión & 16 & 9 & 12 & 5 & 8 & 4 & 16 & 5 & 52 & 23 \\
\hline & 56 & 27 & 40 & 23 & 52 & 28 & 44 & 16 & 200 & 94 \\
\hline
\end{tabular}

Tabla 3

Resumen Total Perfil Innovador

\begin{tabular}{|c|c|c|}
\hline \multicolumn{3}{|c|}{ Total por Tipo de Innovación } \\
\hline Puntaje & Esperado & Obtenido \\
\hline Organización & 56 & 27 \\
\hline Producto/Servicio & 40 & 23 \\
\hline Mercado & 52 & 28 \\
\hline Proceso & 44 & 16 \\
\hline Total & 192 & 94 \\
\hline
\end{tabular}

En las tablas 2 y 3, se observan las diferencias encontradas entre los valores esperados y los obtenidos en el perfil innovador en las áreas de mercado, proceso, producto/servicio y organización, donde se puede observar que la empresa presenta grandes falencias en mercado y en el proceso, ya que no cuenta con recursos ni una infraestructura adecuada para la planeación de procesos de innovación, ni para realizar capacitaciones, y dado a que las nuevas ideas son fomentadas únicamente desde la dirección, no se encuentra aliada la empresa con ningún agente del sistema nacional de innovación y cuenta con un nivel de educación en sus empleados relativamente bajo. Con respecto al proceso las brechas más grandes están en la comunicación, es decir en la planeación estratégica de la organización, ya que para implementar nuevos procesos, cambios de maquinaria y tecnología pertinentes, no se cuenta con una metodología clara para la ejecución de estas decisiones, así lograr reducir la incertidumbre y poder ofrecer a los clientes una mayor confiabilidad a la hora de invertir en sus productos o proyectos con que cuente la empresa.

Revista de Administração e Inovação, São Paulo, v. 12, n.3 p. 310-329, jul./set. 2015. 


\section{CONCLUSIONES}

Los resultados obtenidos de la aplicación de las herramientas TEST CATALAN y ALBACETE indican que en la empresa es preciso que todos sus actores involucrados con la innovación, establezcan una mayor atención y prioricen soluciones en todos los aspectos en los cuales la empresa tiene falencias.

Las brechas obtenidas en el perfil tecnológico de la empresa sugieren que esta debe enfocar sus prioridades en vigilancia tecnológica y en capacitación del personal, la cual representa una de sus más grandes falencias; igualmente en la implementación de software que puedan agregar valor no solo a sus procesos, sino también a sus productos.

La herramienta MGT es muy útil para el diagnóstico de innovación, ya que involucra todas las áreas de una empresa, ayudando al análisis interno en la organización y así mostrar que tan innovadora es esta. También le permite a la empresa ver en donde está fallando y crear estrategias para lograr ser una empresa competitiva frente al mercado.

\section{REFERENCIAS}

Ballón, J. (2008 йил Junio). Identificar la Demanda y Oferta Exportable de los Principales y Potenciales Mercados Internacionales para los Productos del Sector Calzado-La Libertad. Retrieved 2013 йил 6-Marzo from Ministerio de Comercio Exterior y Turismo: http://www.mincetur.gob.pe/Comercio/ueperu/licitacion/pdfs/Informes/4.pdf

Fonseca Rodríguez, S. L., Quintero, J. D., Fúquene, A. M., Saravia, J., Contreras, C. A., Domínguez, K. P., et al. (2011 йил 19,29 у 21-Octubre). Diagnóstico Tecnológico, Herramienta para la Planeación de la Ciencia, la Tecnología y la Innovación. Caso: Cotecmar. Retrieved 2013 йил 8Marzo from XIV Congreso Latino- Iberoamericano de Gestión Tecnológica-ALTEC 2011: http://www.biogestion.unal.edu.co/pdf/articulos/2011_Diagnostico_Tecnologico.pdf

Herrera Piñeros, M. A., Hortúa Leal, S., \& Morales Vendries, А. С. (2009 йил Abril). Estudio de medición de potencial exportador de la empresa de calzado manufactura Hortúa Leal (MHL). $\begin{array}{llll}\text { Retrieved } 2013 \text { йил } & \text { 11-Marzo from }\end{array}$ http://repository.urosario.edu.co/bitstream/10336/1099/1/1020716213.pdf

Zartha Sossa, J. W., Orozco Mendoza, G. L., Vergara Sornoza, J. I., \& Jessie Martínez, D. (2011 йил 23-Agosto). Diagnóstico de Estrategia de Innovación en Grupos de Investigación. Retrieved 2013 
Implementación de herramientas para el diagnóstico de innovación en una empresa del sector calzado en

Colombia

йил 11-Marzo from Journal of Technology Management \& Innovation : www.scielo.cl/pdf/jotmi/v6n3/art15.pdf

Recinos, L. М. (2011 йил Diciembre). Perfil Sectorial de Calzado. Retrieved 2013 йил 16-Marzo from Inteligencia Económica Ministerio de Economía de El Salvador: http://www.tuinventas.com/attachments/article/1574/Perfil\%20Sectorial\%20de\%20Calzado.pdf

CSM3D International Limited. (2011). The Definitive 3D CAD/CAM Shoemaking System. Retrieved 2013 йил 16-Marzo from Shoemaster: http://www.shoemaster.co.uk/

Under Armour. (2013). Prendas de vestir deportivas. Retrieved 2013 йил 16-Marzo from Under Armour: http://www.underarmour.com/shop/us/en/footwear

Salinas, C., \& Angulo, G. (2001 йил Agosto). Comisión Nacional de la Micro y Pequeña Empresa, CONAMYPE. Retrieved 2013 йил 16-Marzo from Estudio del Sub-sector Cuero у Calzado: www.conamype.gob.sv/biblio/pdf/0645.pdf

Fundetec y Anetcom. (2007). Informe de Conclusiones del Primer Taller Nacional de Tecnología aplicadas al Sector Calzado. Alicante.

MUNDIPRESS. (2013). CTCR, la imagen más fiel de la innovación tecnológica en el sector calzado. Revista del Calzado, 16.

OECD y EUROSTAT. (2005). Manual de Oslo, Guia para la recogida e interpretación de datos sobre innovación, Tercera edición. OECD y EUROSTAT.

Ruiz Navas, S., \& Herrera, J. F. (2010 йил 31-Agosto). Gestión de la innovación. Retrieved 2013 йил 17-Marzo from Alianza por la http://www.camaramedellin.com.co/site/Portals/0/Documentos/Memorias/2011/gestion_innovacion -ai.pdf

Fonseca Rodríguez, S. L., Quintero, J. D., Fúquene, A. M., Saravia, J., Contreras, C. A., Domínguez, K. P., et al. (2011 йил 19,20,21-Octubre). Diagnóstico Tecnológico, Herramienta para la Planeación de la Ciencia, la Tecnología y la Innovación. Caso: Cotecmar. Retrieved 2013 йил 08Marzo from http://www.biogestion.unal.edu.co/pdf/articulos/2011_Diagnostico_Tecnologico.pdf

Confederação Nacional da Indústria. (2010). Cartilha: Gestão da Inovação. Retrieved 2013 йил 05 Marzo from http://www.ipdmaq.org.br/Portal/Principal/Arquivos/Downloads/Documentos/DETI/Cartilha\%20G estao\%20Inova\%C3\%A7\%C3\%A3o\%20CNI.pdf

Centro Europeo de Empresas e Innovación. (2013 йил 23-01). ceei ALBACETE. Retrieved 2013 йил 01-Febrero from Auto Diagnostico de la Innovación: http://www.ceeialbacete.com/corps/ceeialbacete/url/autodiagnostico/autodiagnostico.htm

Yoshida Natume, R., Gomes de Carvalho, H., \& de Francisco, А. С. (2008 йил 04). Retrieved 2013 йил 16-Marzo

from http://www.direitoacomunicacao.org.br/index2.php?option=com_docman\&task=doc_view\&gid=39 3\&Itemid=99999999 
Ruiz Navas, S., \& Herrera, J. F. (2010 йил 31-Agosto). Gestión de la innovación. Retrieved 2013 йил 1-Marzo http://www.camaramedellin.com.co/site/Portals/0/Documentos/Memorias/2011/gestion_innovacion _ai.pdf

Herrera Piñeros, M. A., Hortúa Leal, S., \& Morales Vendries, A. C. (2009 йил Abril). Estudio de medición de potencial exportador de la empresa de calzado manufactura Hortúa Leal (MHL). Retrieved 2013 йил 11-Marzo from http://repository.urosario.edu.co/bitstream/10336/1099/1/1020716213.pdf

Instituto Español de Comercio Exterior (ICEX). (2005 йил Marzo). El sector Calzado en . Retrieved 2013 йил 26-Abril from Oficina Económica y Comercial : http://www.icex.es/icex/cma/contentTypes/common/records/mostrarDocumento/?doc=577567

Ortega Cardona, N. C., \& Hurtado Valbuena, C. J. (2012). Procesos de Innovación Aplicado en Empresas de la Industria del Cuero y el Calzado, en la Ciudad de Bogotá. Retrieved 2013 йил 29Abril from http://repository.ean.edu.co/bitstream/10882/844/1/OrtegaNeyla2012.pdf

Centro de Desarrollo Tecnológico para las Industrias del Calzado, Cuero y Afines de Colombia(Ceinnova). (2009). ceinnova.org. Retrieved 2013 йил 06-Mayo from http://www.ceinnova.org.co/asistencia_tecnica.php

Centro Europeo de Empresas e Innovación. (2013 йил 23-01). ceei ALBACETE. Retrieved 2013 йил 01-02 from Auto Diagnostico de la Innovación: http://www.ceeialbacete.com/corps/ceeialbacete/url/autodiagnostico/autodiagnostico.htm

Aguirre Ramirez, J. J. (2010). Metodología para medir y evaluar las capacidades tecnológicas de innovación aplicando sistemas de lógica difusa: caso fábricas de software. Retrieved 2013 йил 02MAYO

Mattos, F., Gastal, C., Rank, L., Emediato, G., Hemán, H., \& Osorio, G. (2008). Kit metodológico para a inovação empresarial. Retrieved 2013 йил 02-Mayo from Movimento Brasil Competitivo: https://www.google.com/webhp?hl=pt\#q=Ferramentas+para+a+gest\%C3\%A3o+da+inova\%C3\%A $7 \% \mathrm{C} 3 \% \mathrm{~A} 3 \mathrm{o} \& \mathrm{hl}=\mathrm{pt} \& \mathrm{ei}=9 \mathrm{RKYUYyEJY}-$

14AOBloGADg\&start=10\&sa=N\&bav=on.2,or.r_qf.\&fp=3f21e0dae69a816f \&biw=1366\&bih=587

Monge González, R., \& Hewitt, J. (2008). Innovación, Competitividad y Crecimiento . Retrieved 2013 йил 02-Mayo from Desempeño de Costa Rica y de su sector de las TICs: http://www.caatec.org/CAATEC/publicaciones/crdigital/CR_Digital_5.pdf 


\title{
IMPLEMENTATION TOOLS FOR THE DIAGNOSIS OF INNOVATION IN A COMPANY OF THE FOOTWEAR SECTOR IN COLOMBIA
}

\begin{abstract}
In this paper an analysis of innovation and technology in footwear sector company, dedicated to producing and selling all kinds of plastic and fabric footwear for women, men and children. Footwear production of synthetic materials such as PVC, has applications in many markets, and provides many opportunities for an organization to be competitive internationally. That is why the company is in need of analysis or current measurement technology resources which employ innovative diagnostic tools (Albacete, Catalan and MTG), presenting the current status of the company as a technology profile and innovative, assessing its competitive position, management and innovation capabilities, then this is an analysis of the results obtained by the tools, which identified the strengths and opportunities that should take the company to improve the technological and innovative, otherwise, create a differentiator in these areas to improve the performance of the company in the market and becoming more competitive in the market.
\end{abstract}

Keywords: Innovation; Technology; Diagnostic tools; Competitiveness.

Data do recebimento do artigo: 06/04/2014

Data do aceite de publicação: 26/03/2015

Revista de Administração e Inovação, São Paulo, v. 12, n.3 p. 310-329, jul./set. 2015. 